\title{
Design and development of three test facilities to evaluate heat transfer performances of advanced and low cost materials and coatings for geothermal application
}

\author{
Paolo Taddei Pardelli ${ }^{*}$, Andrea Mannelli ${ }^{2}$, Claretta Tempesti ${ }^{1}$, Luca Romani $^{2}$,Alberto \\ Baroni $^{2}$, Lorenzo Bosi², Giovanni Ferrara ${ }^{2}$ \\ ${ }^{1}$ Spike Renewables Srl, 50137 Viale Manfredo Fanti 217, Florence, Italy \\ ${ }^{2}$ Department of Industrial Engineering, Università degli Studi di Firenze, 50139 via di Santa Marta 3, \\ Florence, Italy
}

\begin{abstract}
Geothermal energy is accredited as a flexible, controllable and green source of energy.

Heat exchangers (HXs) are one of the most critical components of a geothermal power plant due to corrosion and scaling phenomena. Hence, improvements in the antiscaling and anticorrosion properties as well as heat transfer performance of the HX materials will lead to smaller, more efficient and less costly systems. EU H2020 GeoHex project relies on the use lowcost carbon steel-based material for HXs. Through modifying the surface with nano porous coating and controlling the surface chemistry, the heat transfer performance of single phase and phase change process will be improved.

This paper presents the design and development of three lab scale test rigs to test the effectiveness of innovative materials and superficial treatments on heat exchange with geothermal brine in single, condensing and evaporating phases. The rigs have been equipped with all the necessary instrumentation for control and for data acquisition. In particular, the advanced coatings are applied on a small stainless-steel plate and R134a fluid has been used for heat transfer coefficient characterization in different phase conditions.

GeoHex project has received funding from the European Union's Horizon 2020 research and innovation programme. Grant agreement n.851917.
\end{abstract}

\section{Introduction}

Nowadays, renewable energies play a fundamental role in the challenge to achieve the target of Low-Carbon economy. Geothermal energy is one of the most reliable and stable sources and its development is motivated by the large abundance and distribution of geothermal reservoirs in the world. Thanks to its flexibility, it can play a crucial role in stabilising the electrical grid against fluctuations related to the increasing use of intermittent renewable energy sources such as wind and solar energies [1]. Organic Rankine Cycles

\footnotetext{
* Corresponding author: paolo.taddei@,spikerenewables.com
} 
(ORC) are the most practical technology for generating energy from low and moderate resources and, for this reason, they are frequently coupled with geothermal sources. However, technical challenges due to the corrosive geofluids need to be addressed.

Direct Heat Exchangers (HXs) (e.g. geothermal brine to district heating) and ORC HXs, such as superheater, preheater and evaporator, are in direct contact with the geothermal brine, causing scaling and corrosion at different extent based on the thermophysical condition and chemical composition of the geofluid [5]: these issues cause increased plant maintenance (cleaning) and operational (use of inhibitors) cost and downtime. The limitations of the current heat exchanger materials stem from their reliance on corrosion resistant alloys (CRAs) such as stainless steels or titanium, which are usually recommended to handle aggressive geofluids. These additives:

- Are expensive.

- Have high surface energy: resulting in scale/deposit build up and degradation of performance.

- Have low thermal conductivities resulting in poor heat exchange performance.

For these reasons, HXs in geothermal ORC plants make up a large proportion of the total cost of the plant [6] and therefore improvements or cost reductions in this area would have a huge impact on the overall profitability. The aim of GeoHex project [10], which received funding from the CE H2020 programme GA n.851917, is the development of nanoporous coatings on carbon steel substrates, to contemporary reduce the capital expenditure (CAPEX), thanks to the use of cheaper materials, and improve heat transfer performances, which will lower the exchange surface needed and reduce even more costs.

\section{State of the art and objectives}

Geothermal energy has a high potential to stabilise the thermal and power grid. Currently several factors affect the growth of geothermal plants. These include:

- low overall plant efficiency: to avoid silica scaling, the brine is usually reinjected at a high temperature, hence, full potential of geothermal energy cannot be exploited.

- corrosion and scaling problems [7][8];

- $\quad$ high costs.

Unfortunately, very limited research activities have been done to improve heat transfer performance of the HXs used in the geothermal field. When titanium, CRAs, and stainless steel are used to avoid scaling and corrosion, the HX requires scaling inhibitors and frequent cleaning through hydro-blasting. Furthermore, these are high surface energy materials: as time progresses, their heat transfer performance degrades due to accumulation of scaled/corrosion product. Hence, poor heat transfer performance of standard heat exchangers compels the use of HXs with bigger size, making the CAPEX higher. The utilisation of carbon steels instead of CRAs will allow cheaper heat exchangers to be produced and will also increase their efficiency thanks to higher thermal conductivities [9].

Among the different passive techniques present to enhance heat transfer performance both for single phase and phase change heat transfer process, surface modification seems to be the most effective one. Heat transfer surface can be modified through roughening, texturing, nano structured coating and controlling the surface chemistry. The commonly formed structures using nano structured coatings are nano-porous and nano-finned structures [2]. Generally, nanostructured surface coating is the most preferred method due to the following four reasons [3]: 
- Surface area: the ratio of surface area to volume increases with the decrease in size of the particle. For the same volume of micro structured and nano structured coating, the latter has higher surface area, leading to higher heat transfer.

- Pressure drop: large pressure drop is a major problem for micro channels. Microstructured coating inside the micro-channels would increase the pressure drop even further. However, with nano-structured coating, the thickness is negligible compared with the size of the micro channel.

- Capillary rise: this effect is more dominant in micro and nano-porous structured coating because the small pores can act as capillary tubes.

- Contact time: due to the surface area effect, contact time increases for nano structured coating. Hence, fluid can take more heat from the surface.

For phase change heat transfer, most of the R\&D activities are focused on water and only limited research has been performed with low surface energy fluids, such as ORC working fluids. Heat transfer performance of dropwise condensation (DwC) is several times higher compared to film wise condensation (FC) and similar results occur for nucleate boiling compared to film boiling. To the best of our knowledge, currently, there is no existing robust solution that promotes DwC and nucleate boiling for ORC working fluid HXs. In the field of material research for phase change heat transfer enhancement, it is critical to understand the transition phenomenon i.e. for condensation the transition from drop to film wise condensation and for boiling the transition from nucleate to film boiling, but currently both of them are poorly understood [4]. Phase change heat transfer is difficult to model because it is a transient and multiscale phenomenon involving dynamic motion of multiple interfaces.

Through the development of HX materials, GeoHex project aims to achieve improvements in the antiscaling \& anticorrosion properties, as well as the heat transfer performance, which will lead to smaller, more efficient and cheaper systems. The specific role of the company Spike Renewables is to design and develop three test rigs to test and verify the effectiveness of advanced materials and coatings in the heat exchange performances in single (WP2), condensing (WP3) and evaporating (WP4) phase. In particular, these coatings are applied on 100x100 $\left[\mathrm{mm}^{2}\right]$ thickness $6[\mathrm{~mm}]$ plates which exchange heat between ORC working fluid and geothermal brine (for single-phase case) or water (for phase-change cases) in counter flow arrangement. The heat transfer performance of developed materials will be evaluated by placing these test plates in custom-made HXs: Spike Renewables had to take care of the design of heat exchangers and the test circuit in which they are installed, with all the necessary instrumentation for regulation, control and data acquisition. Partners in the consortium will then perform single-phase, condensing and boiling experiments to evaluate heat transfer performances with 18, 24 and 32 samples respectively, for different use cases materials and coatings. For each of the use cases the same experiment will be performed for 2 identical samples to ensure data reproducibility and hence credibility.

In particular, to enhance the single-phase heat transfer performance nano porous coating will be used and by controlling the surface chemistry and texture the phase change heat transfer (i.e. boiling and condensation) performance will be enhanced. Moreover, for the phase change case, the excellence of GeoHex lies in developing a solution through a combination of numerical solution and image processing algorithm to reveal the droplet/bubble dynamics, mechanism of transition phenomenon occurring in phase change heat transfer and failure modes of induced wettability characteristics. Thanks to these data it will be possible to evaluate the impact of surface morphology (such as roughness, porosity, cavities size and shape, re-entrant structure etc.) and chemistry (wettability) on HTC and droplet/bubble dynamics. The condensation analysis is expected to reveal the transition mechanism of dropwise condensation to film wise condensation and the boiling analysis to 
study the transition mechanism of nucleate boiling to film boiling for low surface tension fluid (refrigerant and hydrocarbon), which will help to develop better condensing and boiling surfaces through surface modification techniques.

\section{Design of the Heat Exchangers \& Test Benches}

The main objective of this study is to enhance heat transfer performances of geothermal fluids by $25-50 \%$ through surface engineering (porous nanostructure) and to increase the antifouling, antiscaling and anticorrosion properties of materials used in heat exchangers for single phase heat transfer application. This will lead to the development of high-performance materials for geothermal application especially for ORC preheater and superheater, where heat from geofluid is transferred to refrigerant/hydrocarbon fluid.

Spike Renewables has taken care of the design and development of the HXs and the lab scale circuits where tests will be performed with all the instrumentation necessary for the operation and control of the plant, together with pressure, temperature and flow sensors for heat transfer evaluation. All sensors are connected to a programmable logic controller (PLC), which is responsible for both the test rig control (start/stop) and data acquisition. In particular, in the P\&IDs of the test rigs, shown in following sections, the red squares drawn in the schemes represent sensors for data acquisition ( $\mathrm{T}$ - temperature, $\mathrm{F}$ - flow, $\mathrm{P}$ - pressure), the black squares represent sensors needed to control the circuit and the black rounds represent points in the circuit for better understanding of the schemes during the description ( $\mathrm{Xr}$ - refrigerant; $\mathrm{Xb}$ - brine; $\mathrm{Xw}$ - water).

As shown in the following specific P\&IDs, several valves with different functions are installed along the circuit. In particular, isolating valves allow to isolate the part of the circuit containing the heat exchanger, facilitating the plate change procedure during tests, without needing to empty all the circuit. The electrical power supply panel is located under the worktop and it is equipped with a main switch with door locking function and a red mushroom-head emergency button with manual reset. Pressing the button the machine and all the powered components immediately stop.

The operator panel is equipped with a 7" touch-screen which allows to start the various processes and monitor them in real time by displaying the flow rate, temperature and pressure parameters in various points of the circuits that make up the test rig equipment as well as allowing the management of the manual regulated components, such as pumps, boiler and cooling chiller.

The test rig is controlled by a programmable logic controller (PLC), placed in the electrical panel, that allows us to measure the physical quantities, like temperature, pressure and fluid flow rate, with a recording frequency of $0.5 \mathrm{~Hz}$. By using a HMI placed in the top of the test bench, PLC can display all the measured quantities in the working circuit and their graphical trend. Through a touch screen display the operator can log the variation of the measured quantities and save it. Through an USB socket the operator can download the saved data.

\subsection{Single-phase test bench (WP2)}

The aim of the single-phase test bench is to evaluate the heat transfer coefficient between an organic fluid and high temperature geothermal brine. The main requirements and critical issues have been:

- brine temperature higher than $200^{\circ} \mathrm{C}$

- High accuracy in the flow rate evaluation

- Fast plate change procedure

- Setting conditions at the inlet of the HX must be maintained constant 
- No contamination between different fluids

\subsubsection{HX Design}

To reach these objectives, a custom-made HX was designed: it is composed of a $154 \times 154 \times 60$ [mm] 316 stainless steel counterflow heat exchanger, designed to meet PN16 pressure standards. It consists of two singular parts joined together by eight M6 stainless steel bolts; between them the coated plate takes place. The spirals of the heat exchanger, manufactured by chip cutting, hold R134a refrigerant fluid in the upper spiral and hot geothermal brine in the lower spiral. Fig. 1 shows the heat exchanger external layout and an internal section: the red component is the exchange plate. The actual built component is shown in Fig. 2. Hydraulic seal between the two sides of the heat exchanger is guaranteed by means of two dedicated O-rings, one for each side. A third O-ring is accommodated between the two stainless steel parts, in order to prevent any further fluid loss. A Swagelok connector, directly welded on the heat exchanger parts, connects the heat exchanger to the WP2 test bench circuit. Stainless steel was used as construction material to avoid corrosion phenomena.
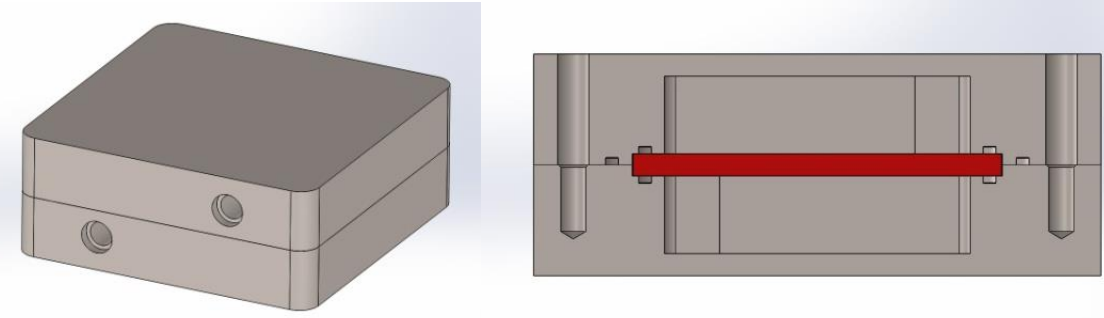

Fig. 1. Single phase HX external layout (left side) and internal section (right side)

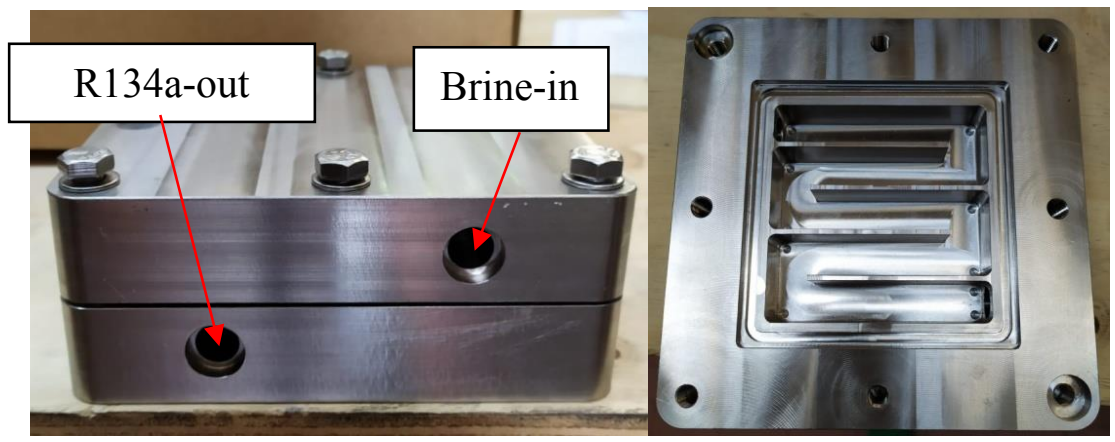

Fig. 2. Single phase HX

The single-phase test rig is composed of three different fluid circuits (Fig. 3): geothermal brine (on the bottom), R134a (in the middle) and refrigerated water (on the top). All the system has been sized considering a 316 stainless steel plate in the HX.

The R134a circuit in liquid phase is represented in pink in the scheme: a mass flow of 42 [L/min] circulates "counter-clockwise" and its temperature increases in the WP2 HX from $25\left[{ }^{\circ} \mathrm{C}\right]$ at the inlet (point $1 \mathrm{r}$ ) to $35\left[{ }^{\circ} \mathrm{C}\right]$ at the outlet (point $2 \mathrm{r}$ ). In point $3 \mathrm{r}$ it enters into a cooler, where the temperature is cooled down again to $25\left[{ }^{\circ} \mathrm{C}\right]$ (point $4 \mathrm{r}$ ). When the test rig is switched on, the pump allows the fluid to circulate through the closed circuit. This pump is lubricated by the fluid itself, so it has a range of frequency out of which it cannot operate; 
in particular it cannot go under $27[\mathrm{~Hz}]$ otherwise the lubrification is not guaranteed. The regulation has been programmed to make the pump work always between 27 and $50[\mathrm{~Hz}]$ : the frequency changes in this range according to the mass flow of the organic fluid required, measured in point $1 \mathrm{r}$. There is also a tank of 22 [L] that supplies the circuit with the organic fluid at the beginning of the experiment.

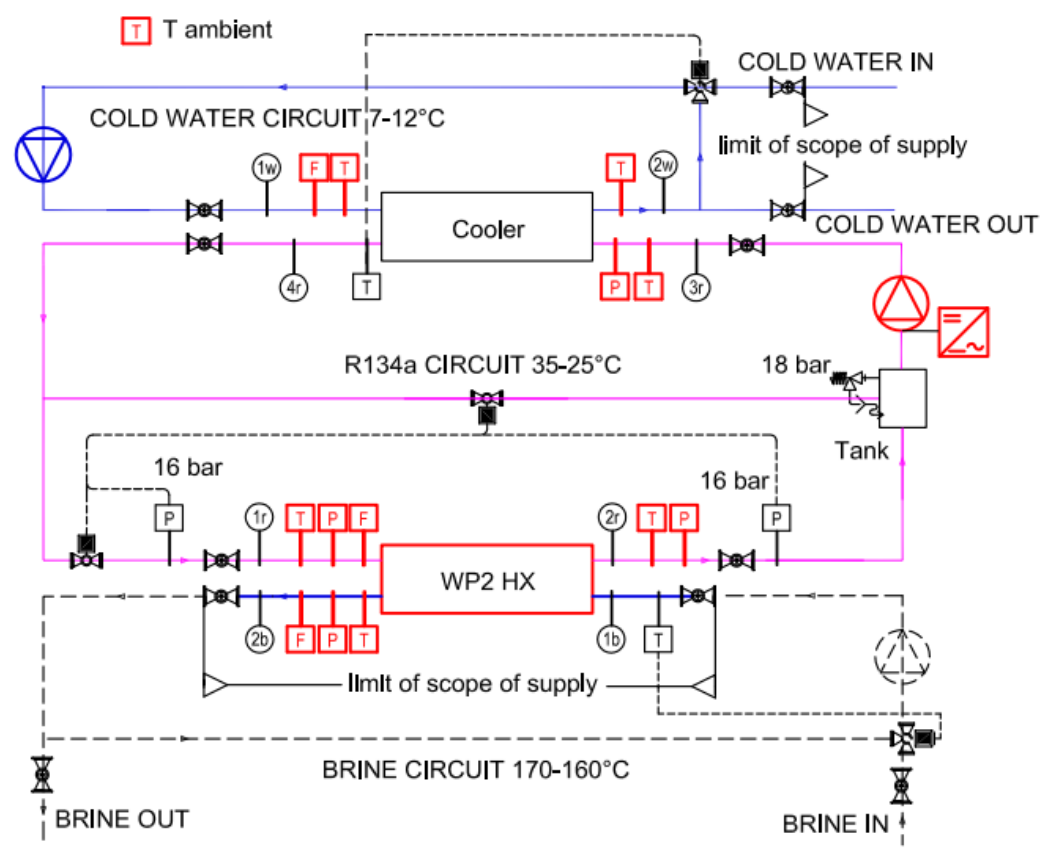

Fig. 3. Single phase test rig layout

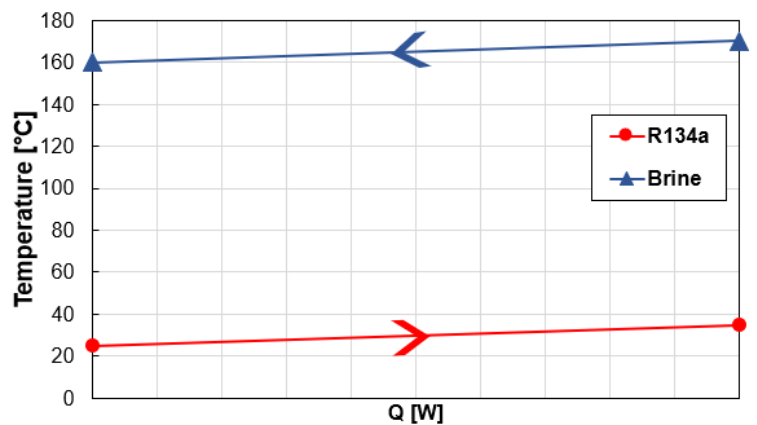

Fig. 4. Single-phase heat exchange graph

The geothermal brine circuit is shown at the bottom of the scheme (Fig. 3). The brine flow of 18 [L/min] enters into the WP2 $\mathrm{HX}$ (point $1 \mathrm{~b}$ ) at $170\left[{ }^{\circ} \mathrm{C}\right]$. A modulating valve allows the mixing of the cooled brine that leaves the WP2 HX at $160\left[{ }^{\circ} \mathrm{C}\right]$ (point $2 \mathrm{~b}$ ) with the hot one at the inlet. By modifying the opening of this valve with a PID control, we can reach the desired temperature of the hot brine at the inlet of the HX (point 1b). After the modulating valve, a pump makes the flow circulate. The design heat exchange plot is shown in Fig. 4.

The refrigerated water circuit is shown in blue on the top of the circuit, and it allows to cool down the organic fluid and to maintain the same inlet conditions at the WP2 HX. The flow of $45[\mathrm{~L} / \mathrm{min}]$, circulating "clockwise" in the scheme, is heated from $7\left[{ }^{\circ} \mathrm{C}\right]$ (point $1 \mathrm{w}$ ) 
to $12\left[{ }^{\circ} \mathrm{C}\right]$ (point $2 \mathrm{w}$ ). As it occurs in the brine circuit, a modulating valve allows the mixing of the hotter water leaving the cooler (point $2 \mathrm{w}$ ) with the cold one at the input. A PID control on this valve allows to maintain the desired set temperature of the organic fluid at the inlet of the WP2 HX (point 1r).

\subsubsection{Experimental layout}

In this section the description of the experimental layout will be carried out. In particular the management system, sensors used and how they are arranged in the test rig.

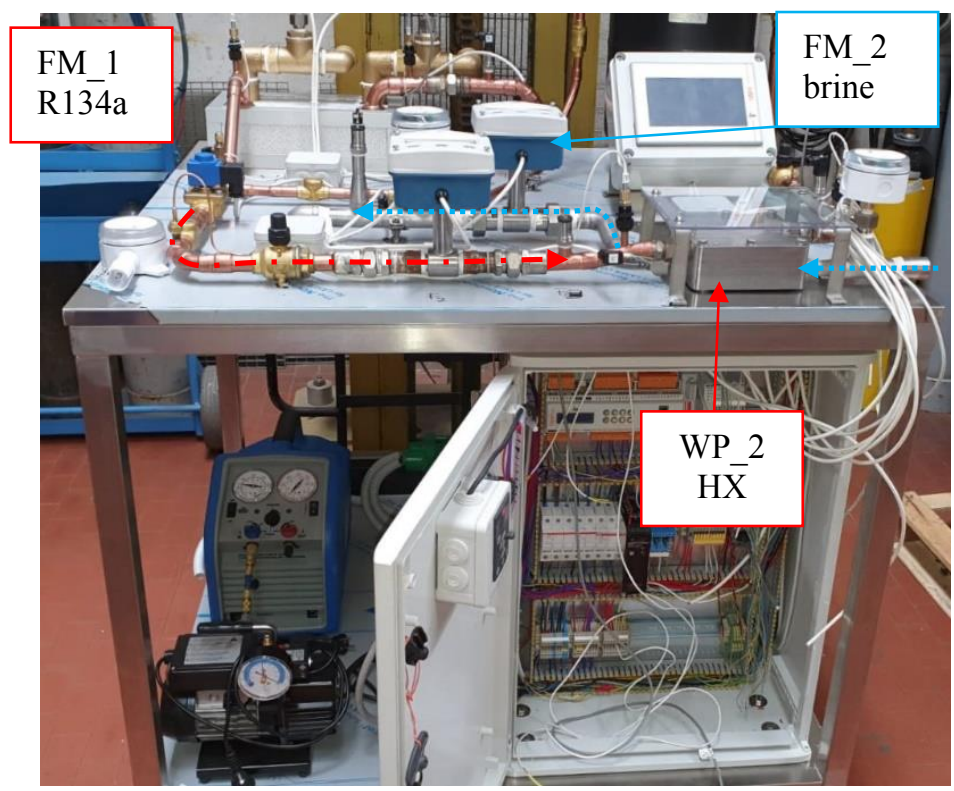

Fig. 5. Single phase test rig

As shown in Fig. 5, the entire test rig is installed on two stainless steel shelves covered by various panels in transparent methacrylate/polycarbonate plastic material. In the upper working surface, the main components of the circuit are placed: sensors, valves, control panel, R134a gas tank and heat exchangers. In the lower surface the rest of the components are located: recirculation pump, electrical panel, vacuum pump, gas recovery unit, etc. The components of the circuit are suitably connected with copper pipes for the gas circuit and in stainless steel for the high temperature circuit: the latter is coated with insulating material to prevent any accidental contact.

The three test rigs designed by the authors follow the construction approach described above; the layout of the circuit has been guided also by encumbrance reasons, so the choice of the instrumentation has represented a big challenge in terms of rangeability, accuracy and dimensions.

In the WP2 test rig, particular attention was paid to the installation of the LX Turbine Flowmeters that are placed both on the R134A side (upstream the HX) and on brine side (downstream the HX). The manufacturer requests 10 diameter straight-duct upstream and 5 diameters downstream of the instrument in order to ensure the maximum accuracy. By referring to Fig. 2, this requirement was respected on R134 side (red dash-dot line) while on brine side (blue dotted line) due to layout constraints the flow meter was installed only five diameters downstream the curve. Nevertheless, during preliminary tests, both flowmeters 
showed a stable and accurate behavior. Pressure and temperature sensors instead, do not need stringent installation requirements.

The R134a in liquid phase flows by a three-stage pump capable of a maximum capacity of $4.14\left[\mathrm{~m}^{3} / \mathrm{h}\right]$ and a nominal absorbed power of $1.5[\mathrm{~kW}]$. This pump is controlled by an inverter that allows to adjust the frequency. At the inlet and outlet of the WP2 HX and at the inlet of the cooler, measuring devices will be installed to monitor the fluid conditions. In particular, by measuring the pressure in points $1 \mathrm{r}$ and $2 \mathrm{r}$ we will be able to know whether some scales have been deposited on the HX surfaces or not. Furthermore, temperature sensors (Pt100 thermistor) and flowmeters will let us know the heat exchanged. The accuracy of the $\mathrm{Pt} 100$ thermistor is $\pm 0.15\left[{ }^{\circ} \mathrm{C}\right]$ at $0\left[{ }^{\circ} \mathrm{C}\right]$. Pressure sensor used in $\mathrm{R} 134$ a circuit have a range from 0.35 to 40 [bar] and an accuracy of $0.25 \%$ FS. The flowmeter has a flow range from 5 to $50[1 / \mathrm{min}]$ and an accuracy $\pm 0.5 \%$ of reading. In the cooling heat exchanger between $\mathrm{R} 134 \mathrm{a}$ and refrigerated water, the same Pt100 thermistor and pressure sensor were installed upstream, as it is shown in point 3r. By measuring the brine mass flow and the temperature before and after the WP2 HX we are able to find the amount of heat exchanged.

The geothermal brine circuit has the same three sensors downstream WP2 HX (point 2b): a flowmeter, a pressure sensor and a Pt100 thermistor. All these sensors can resist the high temperatures of the geothermal fluid $\left(170\left[{ }^{\circ} \mathrm{C}\right]\right)$. In the refrigerated water circuit only upstream and downstream temperature are measured together with water flow rate. Thermistor Pt100 is the same as the other circuit while the flowmeter uses a vortex piezoelectric sensor element. This one has a measuring range between 0.5 and $150[1 / \mathrm{min}]$ and an accuracy of $1 \%$ FS.

\subsection{Phase-change test benches (WP3-WP4)}

Objective of these studies is to develop high-performance material for geothermal ORC condenser (WP3) and evaporator (WP4). The performance of the condensing/boiling surface will be investigated through evaluating heat transfer coefficient (HTC) and capability to sustain dropwise condensation (WP3) and bubble dynamics (WP4). The dynamics of the fluid droplets/bubbles will be analysed by means of physical quantities, like thermal power and heat exchange coefficient, together with a high-speed camera which will provide information about the formation and sliding of droplet fluid (WP3) or the difficulty of detachment of gas bubbles (WP4) comparing different coatings. Consequently, both HXs have to address the following requirements:

- Frontal and side views in order to evaluate visually the heat exchange in a clear way.

- Constant inlet temperature and pressure in the water circuit.

- No contamination between different fluids.

To guarantee high accuracy as well, both test rigs have been designed to maintain constant the organic fluid pressure: the heat exchange allows the fluid to condensate and to boil respectively, without changing its temperature. The fluid circulates in natural convection, without the operation of any pump: the efficiency of the superficial treatments on the exchange plate can be verified and quantified measuring the mass flow of fluid that condensates/boils, keeping all the other parameters constant (pressure, temperature). In this way the mass flow is the measure directly related to the amount of heat exchanged and to the effectiveness of different coatings.

\subsubsection{HX Design}


As previously mentioned, the phase change tests had almost the same requests for both condensing and boiling case: in particular, there was the need to have a clear view of the exchange plate for the development of the numerical solution and image processing algorithm to reveal droplet/bubble dynamics, mechanism of transition phenomenon occurring in phase change heat transfer and failure modes of induced wettability characteristics. For these reasons, the layout is the same for WP3 and WP4 HXs.

These HXs, shown in Fig. 6 and

Fig. 7, are composed of a 197×336x10 [mm] 316 stainless steel condenser unit, designed to meet PN16 standards, characterized by two different parts:

- The upper one is a cylindrical body with 2 glasses which allows to see and capture images about the process.

- The lower one is composed of an internal path where cold water flows to condensate the working fluid.

In the following image the inlet and discharge for the evaporator is shown; for the condenser the path is reversed (Fig. 10).

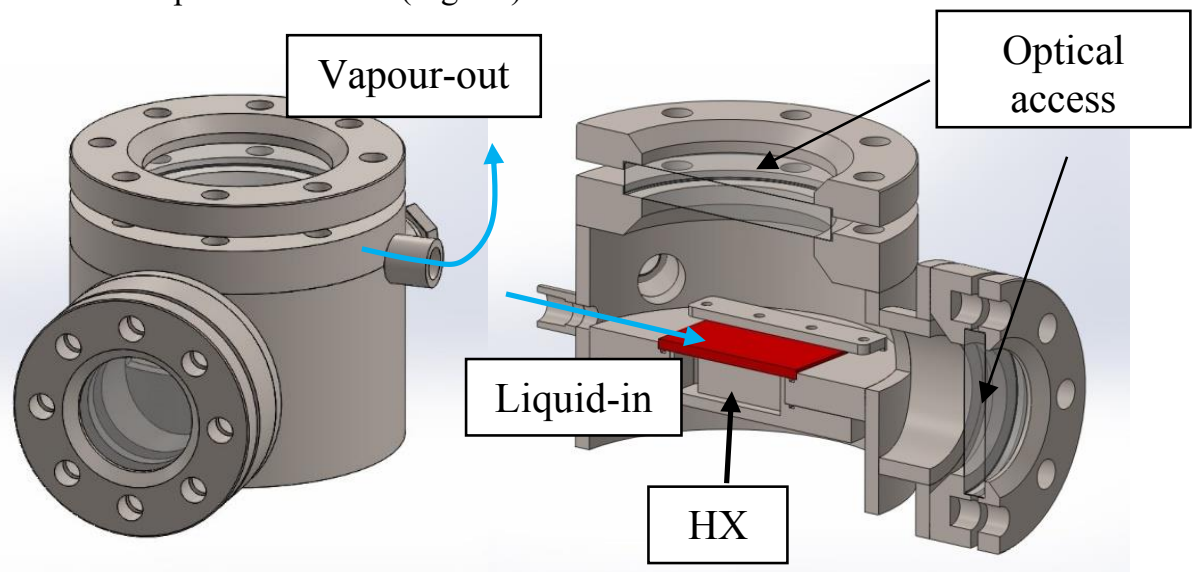

Fig. 6. Phase Change HX external layout (left) and internal section (right); fluid path for the evaporator

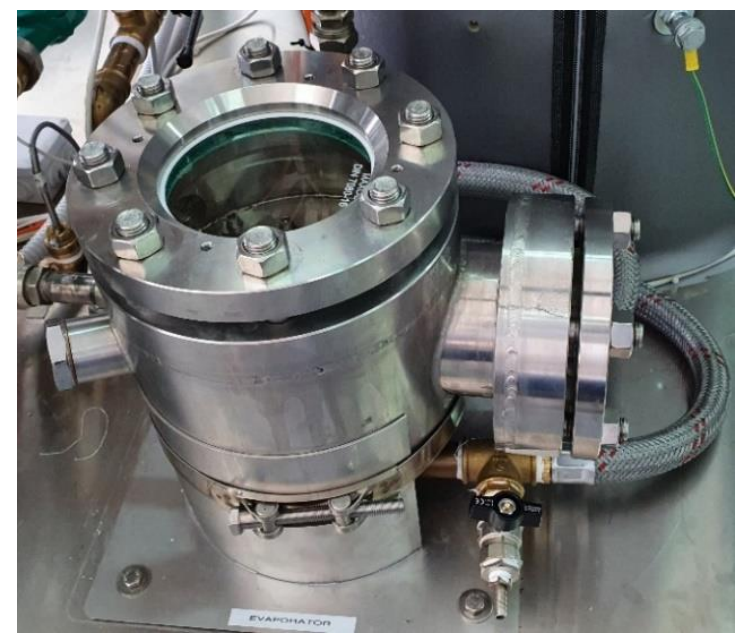

Fig. 7. Phase Change HX 
Between these two parts the exchange plate takes place. Stainless steel was used to avoid corrosion phenomena. Hydraulic seal was ensured by using an O-ring on the bottom of the plate. A DN100 commercial visual glass was installed in front of the coated plate and a DN50 visual glass was placed on the cylinder liner in order to provide a side view of the coated plate. The bottom flanges of these visual glasses were directly welded to the cylindrical body; the upper flange was instead fixed by using eight stainless steel bolts. The borosilicate glasses are interposed between the flanges with two seals. To promote internal visibility and improve the filming of the high-speed camera, two optical accesses for internal lighting were realized in the cylindrical body. It should be noted that the latter was properly designed so as to easily replace the coated plate; in fact, the plate is fixed in working position by means of two retain elements directly screwed to the lower side of the cylindrical element.

The lower part of the cylinder consists instead of a single spiral, in which water flows, directly attached to the lower side of the coated plate. An O-ring was interposed between the cylindrical element and the heat exchanger to avoid fluid leakage. Standard $1 / 4$ hydraulic fittings were used. All connections consist of Swagelok connectors directly welded on the cylinder body.

\subsubsection{Condensing test - WP3}

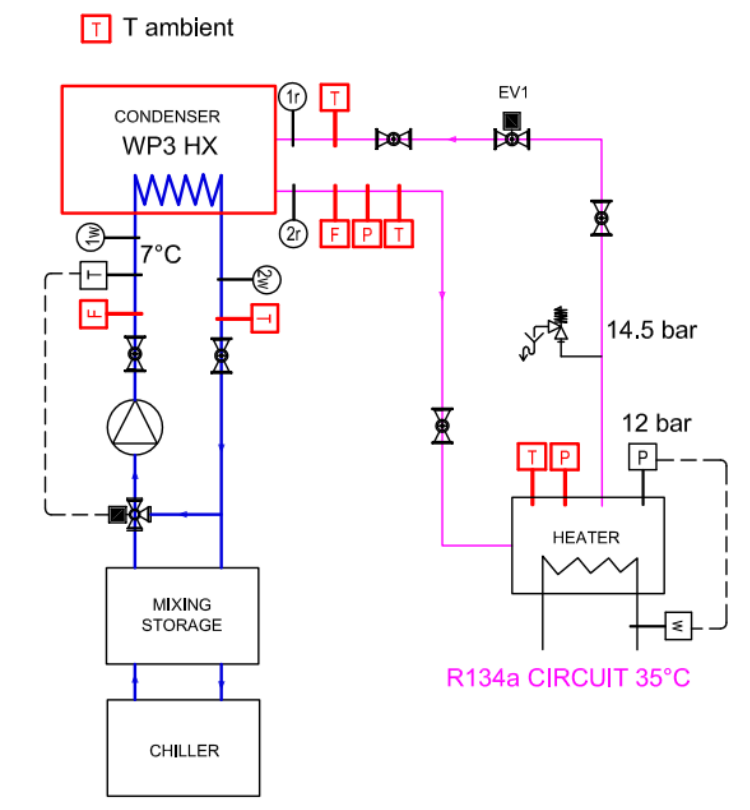

COLD WATER CIRCUIT $7-12^{\circ} \mathrm{C}$

Fig. 8. Condensing test rig layout (WP3)

Unlike the WP2 test case, only two fluids are involved in this circuit. The one on the right in Fig. 8 represents the organic fluid R134a, while the left one represents the cold water used for refrigeration. The organic fluid works in phase change at constant temperature of $35\left[{ }^{\circ} \mathrm{C}\right]$ and the flow rate of $0.26[\mathrm{~kg} / \mathrm{min}]$ flows "counter-clockwise" in the scheme thanks to density variations in natural convection. R134a in gaseous state flows naturally through the WP3 condenser unit (placed in the upper part of the test rig) where it condenses over the coated plate and discharged through the exit hole of cylindrical condenser unit (point $2 r$ ). When the 
fluid is at the lowest part of the rig, it is boiled again thanks to a small tank heated by an electrical resistance. As the temperature of the working fluid doesn't change in the HX, we can evaluate the heat exchanged by measuring the mass flow of the condensate, maintaining the same pressure and temperature values. To keep the same conditions in the circuit, a control on the electrical resistance pressure of the R134a tank has been developed: it is kept constant by switching on/off the electrical resistance. The R134a in liquid state flows through a heater where it is heated up to $35\left[{ }^{\circ} \mathrm{C}\right]$ to return to the gaseous state.

The cold-water circuit is able to condense the organic fluid: it circulates between the WP3 $\mathrm{HX}$ and a chiller, working between 7 and $12\left[{ }^{\circ} \mathrm{C}\right]$ with a flow rate of $4[\mathrm{~L} / \mathrm{min}]$. The water temperature at the WP3 HX inlet (point $1 \mathrm{w}$ ) is set at $7\left[{ }^{\circ} \mathrm{C}\right]$ and thanks to a modulating valve, the cold water out of the chiller can be mixed with the hotter exiting from the WP3 HX (point $2 w)$. Controlling it with a PID we are able to reach and keep the desired set temperature at point 1w. The actual built test rig is shown in Fig. 10.

The design heat exchange plot is shown in Fig. 9.

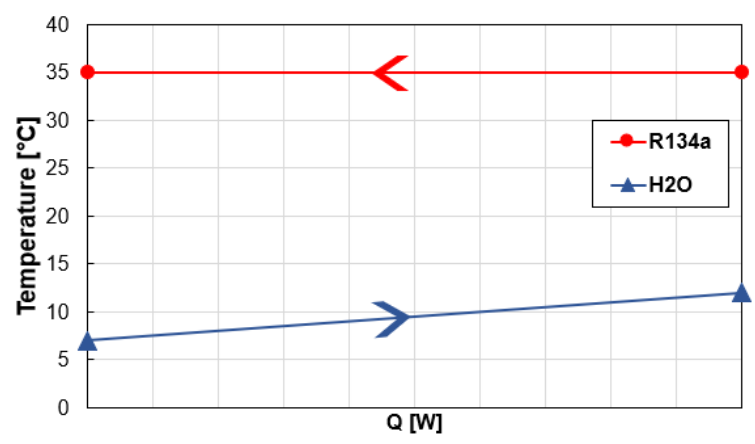

Fig. 9. Condensing heat exchange graph
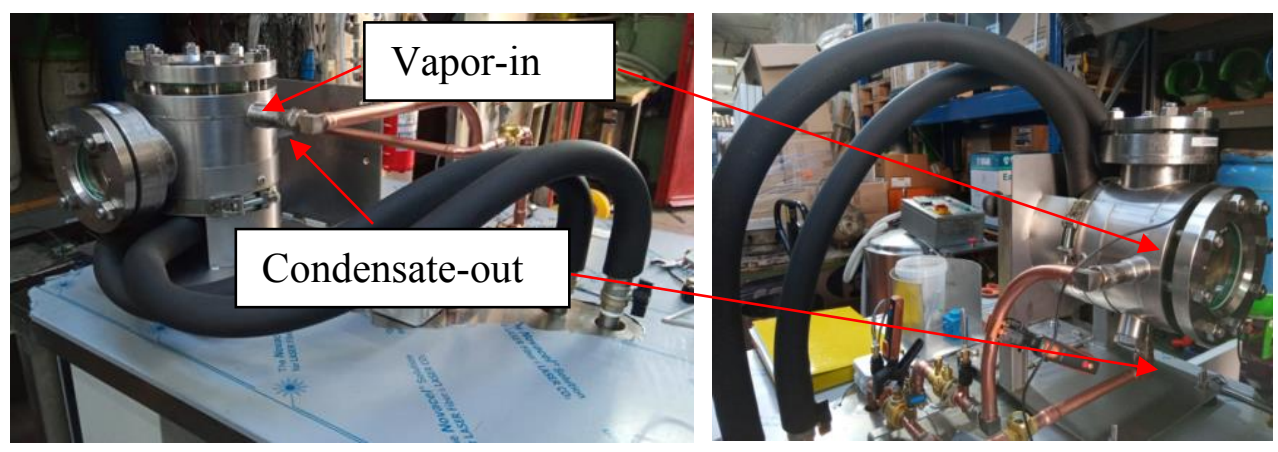

Fig. 10. Positioning of the condensing HX in the test rig with horizontal (left) and vertical (right) plate.

In the condensing unit the experimental setup allows us to perform tests with vertical and horizontal condensing surfaces, evaluating the different slip of the condensate gas over the coated plate.

For the experimental layout, in point 2r, downstream WP3 HX, a pressure sensor, a Pt100 thermistor and a flowmeter have been installed. Upstream the condenser unit (point 1r) a single Pt100 thermistor was installed. These can be used for determining the performance of a coated plate in terms of heat transfer coefficient. Thermistor and pressure sensor have the 
same characteristics of the WP2 test rigs sensor. Instead, the hall-effect flowmeter has flow range between 0.05 and $0.5[1 / \mathrm{min}]$ and an accuracy $\pm 2.5 \% \mathrm{FSD}$.

The flowmeter placed upstream heat exchanger (point $1 \mathrm{w}$ ) has a range between 0.25 and $6.5[1 / \mathrm{m}]$ and an accuracy of $\pm 1.5 \%$ FSD.

\subsubsection{Evaporating test (WP4)}

This unit was designed with the same characteristics of the WP3 pack in terms of materials, dimensions, layout, and pressure requirements. The difference consists in the refrigerant fluid behavior, since the R134a fluid in liquid state fully covers the coated plate and evaporates by means of the heat released from the hot water flowing in the exchanger coil.

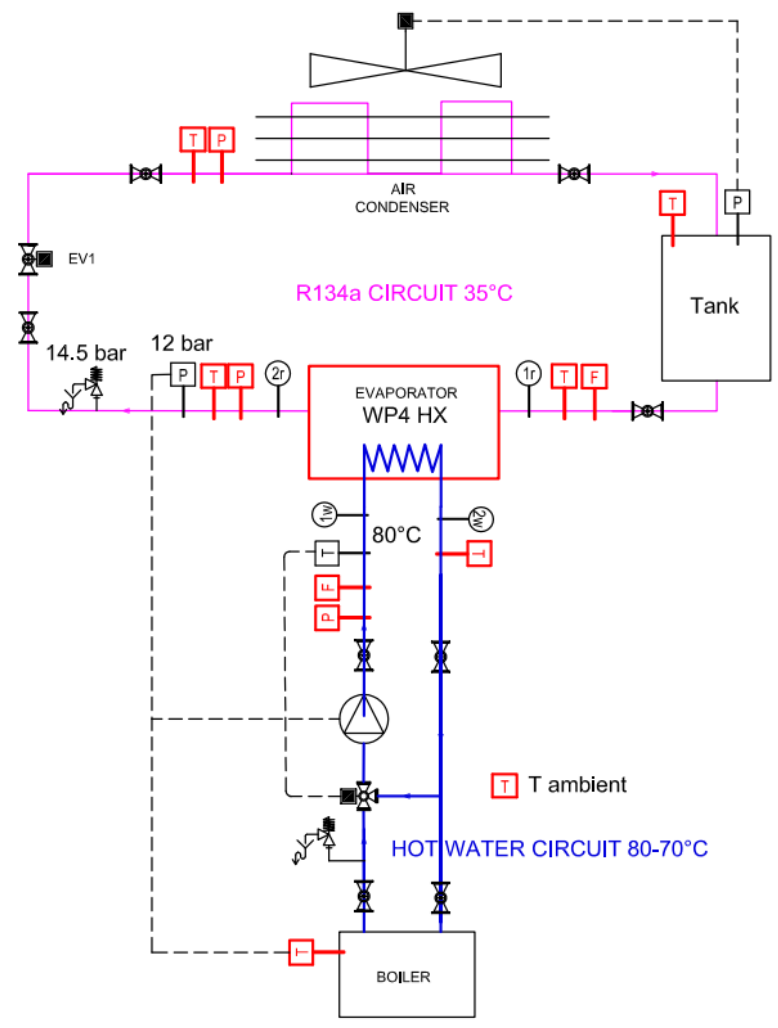

Fig. 11. Boiling test rig layout (WP4)

The diagram shown in Fig. 11 is the WP4 test rig circuit. The pink circuit represents the $\mathrm{R} 134 \mathrm{a}$, while the blue one represents the hot water. The organic fluid works in phase change at a constant temperature of $35\left[{ }^{\circ} \mathrm{C}\right]$. It flows naturally thanks to density variations: it evaporates over the coated plate, placed in the lowest part of the circuit and flows through the exit hole of the cylindrical evaporation unit. The R134a in vapour phase (point 2r) moves then to the upper part of the rig, where it is condensated again thanks to an air condenser. As the temperature of the working fluid does not change in the HX, we can evaluate the heat exchanged by measuring the mass flow, maintaining the same pressure and temperature values. To keep the same conditions in the circuit, a control on the pressure of the R134a tank has been developed: it is kept constant by switching on/off the air condenser. 
The second circuit works with hot water and it includes a commercial boiler and a heat exchanger. The hot water that boils the organic fluid circulates between the WP4 HX and a boiler, working between 80 and $70\left[{ }^{\circ} \mathrm{C}\right]$. The water temperature at the WP4 HX inlet (point $1 \mathrm{w}$ ) is set at $80\left[{ }^{\circ} \mathrm{C}\right]$ : a modulating valve allows the mixing of the hot water out of the boiler with the colder one exiting from the WP4 HX (point 2w); controlling the valve with a PID allows us to reach and keep the desired set temperature at point $1 \mathrm{w}$.

The design heat exchange plot is shown in Fig. 12.

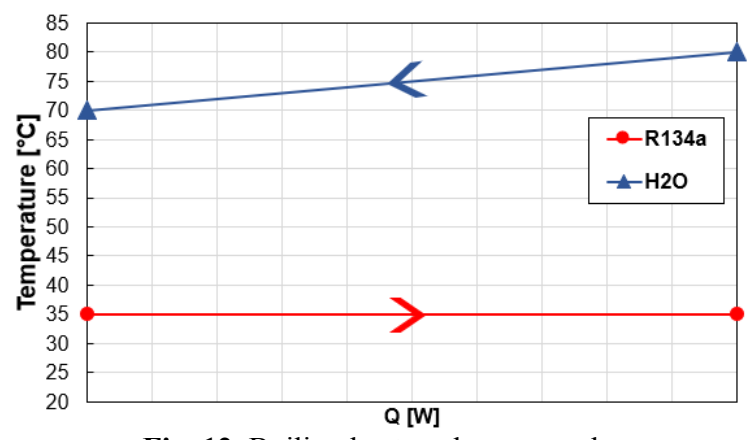

Fig. 12. Boiling heat exchange graph

For the experimental layout, the workbench is equipped with 2 shelves where in the upper surface the air condenser and operator panel are mounted. The actual built test rig is shown in

Fig. 13.

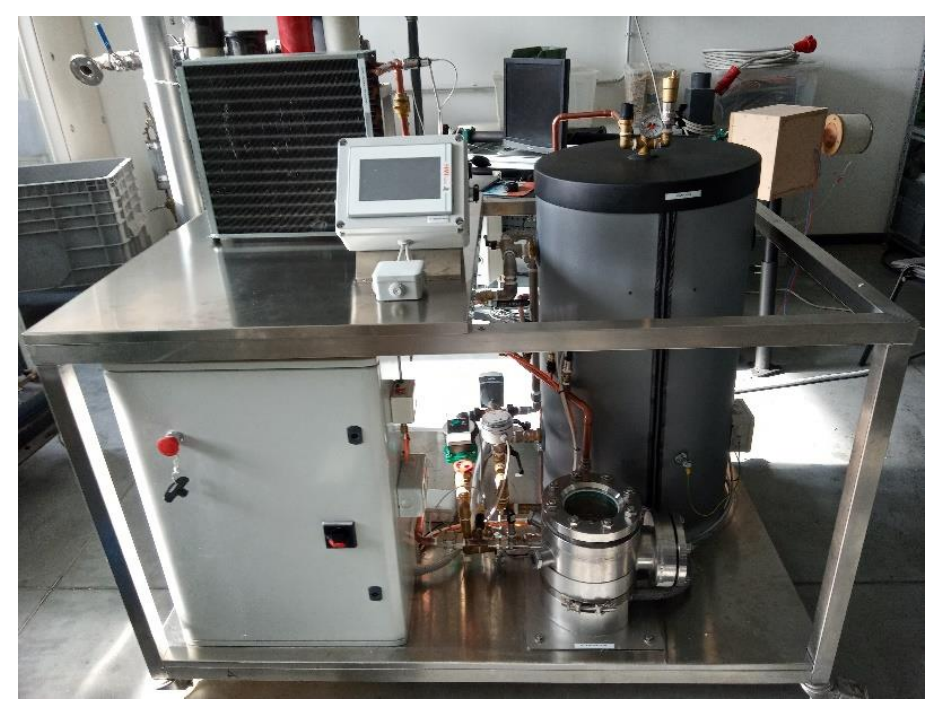

Fig. 13. Boiling test rig

In the lower shelf, the main components of the circuit are placed such as evaporator, boiler, sensors, valves, R134a tank and electrical panel are mounted. Vacuum pump and gas recovery unit are not be fixed. The various components of the circuit are suitably connected with copper pipes for the gas circuit and in stainless steel for the high temperature circuit suitably coated with insulating material to prevent any accidental contact. The test plate of 
$100 \times 100[\mathrm{~mm} 2]$ is placed in the customized HX with two side glass windows to observe flow boiling behavior (i.e., bubble dynamics) using a high-speed camera. Heating rate and temperature of the test block can be controlled by a PID. To measure the inlet and outlet fluid temperature, K-type thermocouples were placed just before the inlet and immediately after the test block. The instantaneous temperatures can be captured by a computerized data taker.

In particular, downstream WP4 HX (point 2r), a pressure sensor and a Pt100 thermistor were placed. Upstream of the WP4 evaporation unit (point 1r), a flowmeter and a Pt100 thermistor were installed. These can be used to determine the performance of a coated plate in terms of heat transfer coefficient. Thermistor and pressure sensor have the same characteristics of the WP2 test rigs sensor. Instead, the hall-effect flowmeter has flow range between 0.05 and $0.5[1 / \mathrm{min}]$ and an accuracy $\pm 2.5 \% \mathrm{FSD}$. The R134a in gaseous state flows through an air condenser and it is stored in a collector. Before the air condenser a pressure sensor and a thermistor were placed in order to check the physical condition of the R134a. Air condenser has a maximum power of $1.1[\mathrm{~kW}]$.

\section{Test Benches Commissioning}

In the GeoHex project, the design and the construction of the test benches for heat exchange performance evaluation is the first step of many activities in which lots of industrial and academic partners are involved. For this reason, results on future tests on the innovative materials are not yet available. However, commissioning tests have been made in order to control and validate the consistency of the test benches. One of the peculiarities of these test benches is the possibility to calculate indirectly the heat transfer coefficient by basic thermodynamic formula of heat exchanger. The accuracy is guaranteed by precise sensors installed in the immediate vicinity of the test rig. In the following images there are some examples of the record of the main operating parameters. In

Fig. 14a and in

Fig. $14 \mathrm{~b}$ the trends of flow rate and temperature are represented respectively.

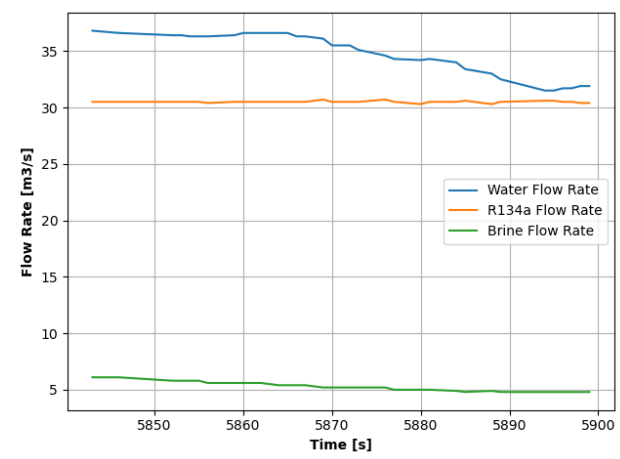

a)

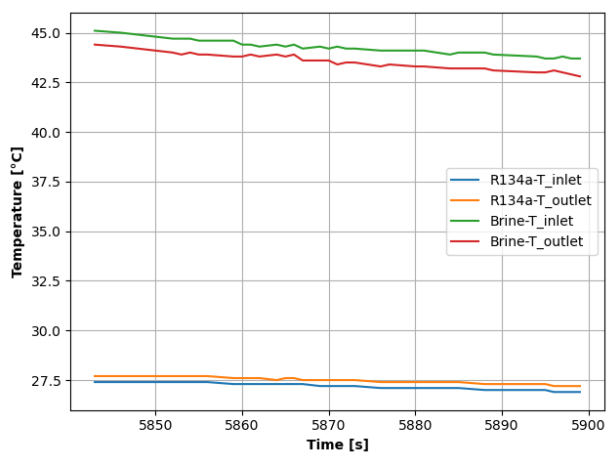

b)

Fig. 14. a) Flow rate recording in WP2 b) Temperature recording in WP2 


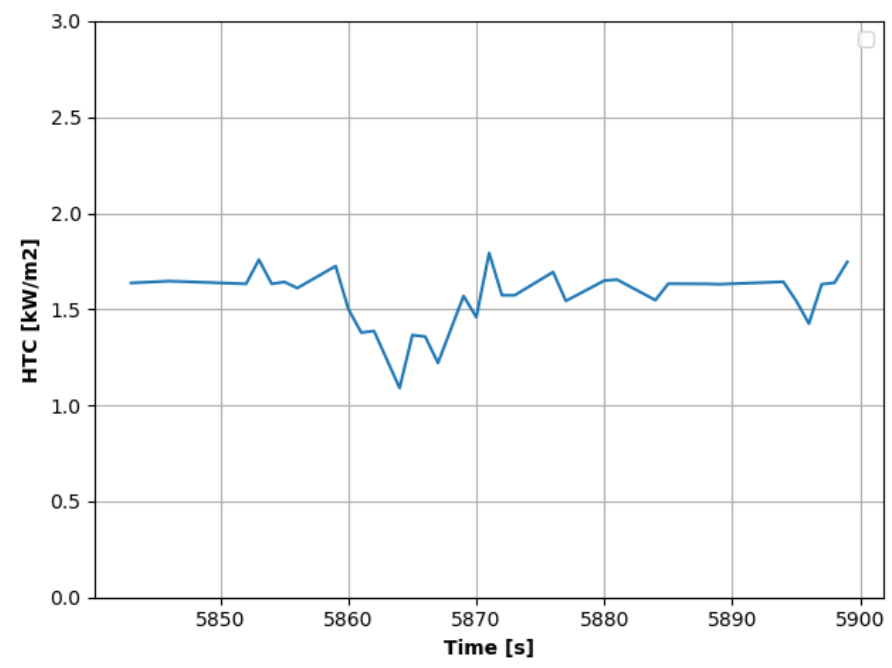

Fig. 15. WP2 Heat Transfer Coefficient

By thermodynamic relations, it is possible to evaluate the trend of the Heat Transfer Coefficient of the HX. As shown in Fig. 15, the test rig is able to maintain a steady HTC, permitting to have a stable data collection for a long period.

The image in Fig. 16 shows a framework of the formation of bubbles on the plate in the evaporating test rig WP3. The commissioning has shown that test benches address the objectives with the required accuracy for the next steps of the GeoHex project.

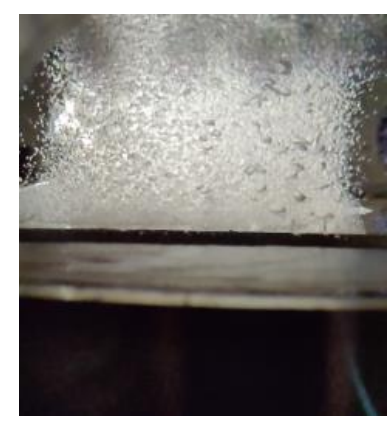

Fig. 16. Bubble formation in the preliminary tests

\section{Conclusions}

In this paper, it has been presented the design and development of three lab scale test rigs to test the effectiveness of low-cost carbon steel-based materials and superficial treatments on heat exchange with geothermal brine in single, condensing and evaporating phase. The rigs have been equipped with all the necessary instrumentation for control and for data acquisition of the operational parameters. In particular, the advanced coatings are applied on a small stainless-steel plate of $10 \times 10 \mathrm{~cm}$ and R134a fluid has been used for heat transfer coefficient characterization in different phase conditions. The single-phase test rig allows to evaluate the heat exchanged between the organic fluid R134a and the on-site geothermal brine at $220^{\circ} \mathrm{C}$. The phase change test rigs (one in condensing and the other in evaporating phase), thanks to the presence of sight glasses in the heat exchanger layouts, have the 
peculiarity to make visible the bubbles formation on the exchange plates. Connecting a specific camera to the evaporating rig enables the measurement of the bubbles coalescence and it allows to find a relation between heat exchanged and bubbles growth. The commissioning of test benches has demonstrated the capability to accurately evaluate all the parameters required to address both the enhancements in the antiscaling \& anticorrosion properties with the aim to maximize the heat transfer reducing dimensions and costs of HX.

In the next two years, the other industrial and academic partners involved in GeoHex project will perform on these test benches all the activities related to the characterization of materials properties.

\section{ACKNOWLEDGMENTS}

The Project has received funding from the European Union's Horizon 2020 research and innovation programme under grant agreement $\mathrm{N}^{\mathrm{o}} 851917$.

http://geohexproject.eu/
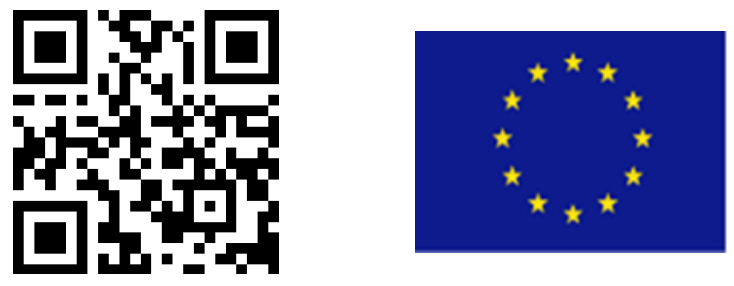

\section{GEOHEX}

\section{References}

1. S. B. Bina, S.Jalilinasrabady, H. Fujii, Energy 140 Part 1, 1096-1106 (2017)

2. S. Kalaiselvam, M: S. Gugan, E. Kuraloviyan, R.Meganathan, A.Niruthiya Priyan, M. R. Swaminathan, Journal of Thermal Science, 18, 358 (2009)

3. Sujith Kumar C. S, S. Suresh, Rajiv K., International Journal of Scientific \& Engineering Research, 3 Issue 6 (2012)

4. D. Attinger, C. Frankiewicz, A. Betz, T. Schutzius, R. Ganguly, A. Das, C. Megaridis, MRS Energy \& Sustainability, 1, E4 (2014)

5. F.Ceglia, A.Macaluso, E.Marrasso, M.Sasso, L.Vanoli, Energies (2020), 13, 2737

6. S.L.Gómez Aláez, P.Bombarda, C.M.Invernizzi, P.Iora, P.Silva, Applied Energy 154 (2015), 882-890

7. S.J.Zarrouk, B.C.Woodhurst, C.Morris, Geothermics 51 (2014), 445-459

8. M.Tan, R.Karabacak, M.Acar, Geothermics 62 (2016), 70-78

9. A.Buzaianu, P.Motoiu, I.Csaki, A.Ioncea, V.Motoiu, Proceedings 2(23), 2018, 1434

10. https://www.geohexproject.eu/ 